\title{
SU UMILI E CULTURA FRA OTTO E NOVECENTO
}

\author{
IVAn Montebugnoli \\ Liceo Scientifico «E. Fermi» - Bologna \\ ivan.montebugnoli@hotmail.it
}

\section{RESUMEN}

La relación entre los humildes y la cultura es un tema del que tratan Los novios de Manzoni, Los Malavoglia de Verga así como I fuochi del Basento de Raffaele Nigro. Las dos últimas novelas están ligadas también a la llamada cuestión meridional.

Palabras Clave: humildes, cultura, Manzoni, Verga, Nigro.

\section{RIASSUNTO}

Il rapporto fra gli umili e la cultura è un tema comune ai Promessi Sposi di Manzoni, ai Malavoglia di Verga e ai Fuochi del Basento di Raffaele Nigro. Questi due ultimi romanzi affrontano anche il problema della cosiddetta questione meridionale.

Parole chiave: umili, cultura, Manzoni, Verga, Nigro.

\section{MANZONI E GLI UMILI}

È noto come, attraverso la non nuova trovata del «dilavato e graffiato autografo» del Seicento, già nell'Introduzione ${ }^{1}$, il Manzoni dichiari di porre al centro della sua opera gli

1 Alle pp. 949-51 di MANZONI, Alessandro (1973): Tutte le opere, a c. di M. Martelli, Firenze, Sansoni, 2 voll., da cui, salvo diversa indicazione, sono tratte tutte le citazioni manzoniane. 
umili, le «gente meccaniche e di piccol affare», una coppia, se non reale verosimile, di umili filatori di seta, Renzo e Lucia. Essa nell'economia del romanzo in tutti i sensi si contrappone a una «coppia d'alto affare», quella di don Ferrante e donna Prassede, emblematica di quei «Prencipi e Potentati, e qualificati Personaggi» sui quali unicamente si concentra l'attenzione dell' Historia con l' $H$ maiuscola.

Ora, che la storiografia ufficiale taccia degli umili, dei «vinti», Manzoni, amico e frequentatore degli idéologues francesi, Cabanis, Tracy, Fauriel, lo sa bene; e memorabile è la formulazione che di questo concetto egli dà nel Discorso sopra alcuni punti della storia longobardica in Italia:

Un'immensa moltitudine d'uomini, una serie di generazioni, che passa sulla terra, sulla sua terra, inosservata, senza lasciarci traccia, è un triste ma importante fenomeno (p. 1981).

È così che, dopo avere inevitabilmente parlato nelle tragedie di conti, capitani di ventura e di prìncipi e re franchi e longobardi, relegando semmai la considerazione delle masse anonime, «volgo disperso che nome non ha», ai cori «cantucci lirici», all'altezza del romanzo il Manzoni decide, in maniera rivoluzionaria per l'Italia, di concentrare la sua attenzione su una coppia di umili, una "rivoluzione" concettuale a cui quasi inevitabilmente se ne affiancherà una linguistica, l'impiego di una lingua non più depositata nei vocabolari, ma viva ed effettivamente parlata: secondo la sua nota formula, il «fiorentino parlato dalle persone colte».

Ma egli non si accontenta di portare gli umili sulla ribalta della letteratura italiana, fa di essi anche i portavoce della sua ideologia e delle sue più profonde convinzioni religiose e morali. Rileggiamo il finale dei Promessi Sposi (p.1276). Nell'apparente idillio finale $^{2}$, Renzo e Lucia riflettono sulle loro passate peripezie. Renzo dice di avere fatto tesoro degli errori passati:

Ho imparato [...] a non mettermi ne' tumulti: ho imparato a non predicare in piazza: ho imparato a guardar con chi parlo: ho imparato a non alzar troppo il gomito: ho imparato a non tenere in mano il martello delle porte, quando c'è lì d'intorno gente che ha la testa calda: ho imparato a non attaccarmi un campanello al piede, prima d'aver pensato quel che ne possa nascere.

Lucia però non è del tutto soddisfatta di questa «dottrina»e un bel giorno ribatte al suo «moralista»:

e io [...] cosa volete che abbia imparato? Io non sono andata a cercare i guai: sono loro che son venuti a cercar me.

Da una sintesi delle due diverse opinioni deriva la conclusione che il Manzoni decide di riportare come «il sugo di tutta la storia»:

i guai vengono bensì spesso, perché ci si è dato cagione, ma [...] la condotta più cauta $\mathrm{e}$ più innocente non basta a tenerli lontani, e [...] quando vengono, o per colpa o senza colpa, la fiducia in Dio li raddolcisce, e li rende utili per una vita migliore.

2 Si veda su questo almeno RAIMONDI, Ezio (2000): Il romanzo senza idillio, Torino, Einaudi, pp. 18589 e 305-07, e FORTI, Fiorenzo (1981): «Manzoni e il rifiuto dell'idillio», in Lo stile della meditazione, Bologna, Zanichelli, pp. 185-88. 
Nonostante il tipico understatement manzoniano, credo che abbia ragione FORTI (1981: 185-88) nel sottolineare il sottofondo teologico, di stampo giansenistico, delle semplici considerazioni dei due sposi: insomma, alla base di «questa conclusione, benché trovata da povera gente» ci sono tutte le riflessioni morali e religiose che il Manzoni ha maturato sui moralistes francesi del grand siècle. Così facendo, il Manzoni realizza l'ideale di un'arte popolare che in Italia altri vanamente inseguivano.

Ben diverso il trattamento che egli riserva alla «coppia d'alto affare» don Ferrante e donna Prassede, significativamente destinata ad essere vittima di quella peste che verrà invece vinta e superata dai due umili protagonisti. Tralasciando l'ironia impietosa riservata a donna Prassede, il cui «studio era di secondare i voleri del cielo: ma faceva spesso uno sbaglio grosso, ch'era di prender per cielo il suo cervello» (p. 1160), è qui più utile prendere in considerazione la figura di don Ferrante, verso il quale l'ironia del Manzoni non è comunque meno tagliente. Celebre la descrizione, nel cap. XXVII, della biblioteca di don Ferrante, rappresentazione di un sapere erudito e fine a se stesso, scollegato dalla vita, e per questo privo di interesse, tanto che, osserva il Manzoni, verosimilmente smembrata dopo la morte del suo proprietario, essa «è forse ancora dispersa su per i muriccioli» (p.1267). Né si dimentichi che il «colto» don Ferrante muore, dopo avere sillogisticamente «dimostrato» che la peste non esiste:

His fretus, vale a dire su questi bei fondamenti, non prese nessuna precauzione contro la peste; gli si attaccò; andò a letto, a morire, come un eroe del Metastasio, prendendosela con le stelle (p. 1267).

Da un lato, dunque, due giovani semianalfabeti giungono a conclusioni filosofico-religiose che il Manzoni sottoscrive, poiché sono le sue, dall'altro la cultura libresca di don Ferrante è ridicolizzata e serve al suo depositario per negare l'evidenza della realtà.

Quanto detto finora si colloca sul piano della genesi stessa dei Promessi Sposi e della sua economia strutturale. Ma è interessante anche osservare che molto spesso nel corso del romanzo la cultura si configura quale strumento di oppressione nei confronti degli umili, anziché, come dovrebbe essere, di loro elevazione sociale e spirituale: «Così va spesso il mondo» conclude con disincanto il Manzoni a proposito di Renzo, il quale, nella «notte degl'imbrogli e de' sotterfugi», entrato con l'inganno in casa di don Abbondio, lo tiene assediato in una stanza e «ha tutta l'apparenza d'un oppressore; eppure, alla fin de' fatti, era l'oppresso» (p. 1010). Oppresso, possiamo aggiungere, da una cultura snaturata rispetto al suo vero fine, e che spesso si avvale di uno strumento linguistico incoprensibile agli umili, il latino, o forse meglio il latinorum.

Intimorito dai bravi di don Rodrigo, il povero don Abbondio, che già «non era nato con un cuor di leone» (p. 956), e che in questo caso non ha tutti i torti a temere per la sua vita $^{3}$, cerca di guadagnare tempo e comincia a trovare scuse per rimandare il matrimonio. A compimento della sua strategia, egli comincia a snocciolare gli «impedimenti dirimenti» come li ha appresi in seminario: davvero.

${ }^{3}$ A ragione il Pirandello nel saggio sull'Umorismo avverte che don Rodrigo le schioppettate le dava per 
«Error, conditio, votum, cognatio, crimen, Cultus disparitas, vis, ordo, ligamen, honestas, Si sis affinis,...» (p. 962)

fino a che Renzo perde la pazienza ed esclama la famosa frase: «Che vuol ch'io faccia del suo latinorum? $\gg^{4}$. Ma intanto il curato è riuscito a convincere l'inesperto giovane che egli ne sa più di lui e che il matrimonio deve essere rimandato.

E ancora il latino ricorre nel banchetto nel palazzotto di don Rodrigo, dove, seduti attorno alla stessa tavola, si trovano, oltre a due

convitati oscuri [...] che non facevano altro che mangiare, chinare il capo, sorridere e approvare ogni cosa che dicesse un commensale, e a cui un altro non contraddicesse (p. 986),

il padrone di casa, il cugino, conte Attilio, l'Azzecca-garbugli e «il signor podestà, quel medesimo a cui, in teoria, sarebbe toccato a far giustizia a Renzo Tramaglino, e a fare star a dovere don Rodrigo» (ibidem). Ed ecco che i feciales, i sacerdoti romani incaricati di recare le dichiarazioni di guerra, vengono dall'ignorante conte Attilio confusi con gli «ufiziali degli antichi Romani»; e che l'Azzecca-garbugli, per elogiare il vino dell'anfitrione, «l'Olivares de' vini», esordisce «censui, et in eam ivi sententiam» (p. 990), provocando con quello che segue una discussione sull'imminente carestia, di cui però i ricchi benestanti e i loro conniventi scagnozzi non conoscono le terribili conseguenze, che invece già si cominciano a far sentire fra i poveri ${ }^{5}$.

Ma torniamo alla figura del dottor Azzecca-garbugli, l'avvocato a cui Renzo si rivolge, su consiglio di Agnese, nell'ingenua speranza di ricevere aiuto legale contro i soprusi di don Rodrigo. Il dottore, però, che ha scambiato Renzo per un bravo, per dimostrargli la gravità della situazione di chi minaccia un curato perché non celebri un matrimonio, gli sciorina davanti una «grida fresca» (p. 970) che contempla il caso, mentre Renzo, lo ripetiamo, semianalfabeta, «gli andava dietro lentamente con l'occhio» (p.971). Poi gli espone chiaramente la sua idea di giustizia: «a saper ben maneggiare le gride, nessuno è reo, e nessuno è innocente» (p. 972). Infine, quando Renzo chiarisce di non essere affatto un bravo, ma una vittima di don Rodrigo, non solo lo caccia in malo modo ma gli fa addirittura restituire i quattro capponi che Renzo gli ha portato in dono. In conclusione, l'abilità e la cultura professionali dell'avvocato imbroglione e corrotto sono sempre e solo al servizio del più forte.

Continuando nella rassegna del latino come espressione di una cultura e di un potere congiurati ai danni degli umili, accenniamo solo tra parentesi al paolino Omnia munda mundis («tutto è puro per i puri») con cui fra Cristoforo riesce a convincere fra Fazio, il laico sagrestano del convento di Pescarenico, ad accogliervi di notte due donne, Agne-

${ }^{4}$ Una battuta che ha avuto tra l'altro la forza di aggiungere un nuovo termine al vocabolario italiano, il latinorum, a indicare la lingua latina, e forse qualsiasi altra lingua, usata in modo volutamente pedantesco e incomprensibile.

5 Memorabili fra tutte l'immagine della «fanciulla scarna» che «tenendo per la corda al pascolo la vaccherella magra stecchita, guardava innanzi, e si chinava in fretta a rubarle, per cibo della famiglia, qualche erba, di cui la fame aveva insegnato che anche gli uomini potevan vivere» (p. 976), e quella, a casa di Tonio, della polenta, la cui mole «era in ragion dell'annata, e non del numero e della buona voglia de' commensali», tanto che, una volta versata sul tagliere «parve una piccola luna, in un gran cerchio di vapori» (p. 996). 
se e Lucia, «dimenticando che questo non intendeva il latino»: impiegato qui a fin di bene, salvare due innocenti dagli artigli di don Rodrigo, si tratta pur sempre di un latino che soggioga l'incolto col suo «senso misterioso» (p. 1016).

Ben più significativo ai nostri fini è lo spagnolo del gran cancelliere Antonio Ferrer, il quale, durante i tumulti milanesi di San Martino, arriva in carrozza a salvare il vicario di provvisione dalla furia della folla inferocita, che lo ritiene responsabile della fame in città. Il narratore pensa invece che Ferrer abbia indirettamente provocato quella rivolta popolare, poiché ha in precedenza fissato un prezzo politico («meta») per il pane, insostenibile per i fornai, e quindi commenta che «veniva a spender bene una popolarità mal acquistata» (p. 1059), cioè a salvare la vita di un uomo, anche se per mezzo della demagogia. Ferrer avanza in carrozza e dispensa alla folla sorrisi e slogan in italiano («pane e giustizia», p. 1063), mentre a bassa voce esprime in spagnolo i suoi sentimenti più intimi e le sue reali intenzioni: egli viene a condurre in prigione il vicario e a "dargli il giusto gastigo che si merita", ma súbito dopo aggiunge sottovoce: "si es culpable" (p. 1061). Renzo, contrario in ogni modo all'idea dell'omicidio, si schiera subito dalla parte di Ferrer, che è per lui quello «che aiuta a far le gride», ricordandosi del «vidit Ferrer» (ancora il latino!) che l'Azzecca-garbugli gli ha fatto vedere in calce alla grida del cap. III (p. 971, v. supra), e con le sue spalle da montanaro contribuisce non poco a fare avanzare la carrozza tra la folla.

Eppure, per quanto «invaghito» della «decorosa vecchiezza» di Ferrer (p. 1062), a Renzo, ormai ubriaco all'osteria della luna piena, sembra che anche il gran cancelliere abbia usato «qualche parolina in latino ... siés baraòs trapolorum» (p. 1072), dove l'ultima, immaginaria parola sembra formata per analogia con il latinorum, e tutta la frase esprime la diffidenza di Renzo «povero figliuolo» verso il mondo dei «signori», i quali,

quando vogliono imbrogliare un povero figliuolo [...] e s'accorgono che comincia a capir l'imbroglio, taffete, buttan dentro nel discorso qualche parola in latino, per fargli perdere il filo, per confondergli la testa (p. 1070).

Invece, tutto andrebbe fatto «in volgare, e senza carta, penna e calamaio», tanto che egli rifiuta di dare all'oste «nome, cognome e patria» (p. 1069), mettendosi così definitivamente nei guai con la giustizia. Verrebbe quasi da esclamare: in vino veritas!

Certo è difficile dare torto a Renzo sull'uso vessatorio del latino da parte delle autorità, se anche solo si pensa che in latino è redatto il dispaccio con cui il «signor capitano di giustizia», dà ordine al «signor podestà di Lecco» (quello stesso che siede a tavola con don Rodrigo!) di prendere informazioni su Lorenzo Tramaglino, ad ogni buon conto «facta debita diligentia» nella casa (o meglio domus) «praedicti Laurentii Tramaliini»; il che significa, come postilla Manzoni, «si fa come in una città presa d'assalto» (pp. 1097-98). Un Lorenzo Tramaglino «reo buon uomo» (p. 1076), che la giustizia, non potendo o non volendo colpire i veri colpevoli, indica come «uno de' capi» dei tumulti di San Martino, secondo il racconto straniante e "reazionario" che della rivolta popolare fornisce il mercante di Milano agli oziosi avventori dell'osteria di Gorgonzola (pp. 1086-89).

Ma il latino è la lingua non solo di una giustizia iniqua, bensì anche di politici insipienti, che si disinteressano delle condizioni e delle sofferenze degli umili, preoccupandosi unicamente del proprio prestigio e della guerra. Così, nell'imminenza della discesa 
dell'esercito imperiale di Ferdinando II, che si teme possa diffondere la peste tra la popolazione, il primo governatore di Milano, il «Signor Gonzalo Fernandez di Cordova» afferma, in volgare:

che i motivi d'interesse e di riputazione, per i quali s'era mosso quell'esercito, pesavano più che il pericolo rappresentato; che con tutto ciò si cercasse di riparare alla meglio, e si sperasse nella Provvidenza (p. 1188).

Addirittura in latino è invece la risposta del suo successore, il genovese Ambrogio Spinola, ai delegati del tribunale di sanità che lo informano che la peste si è ormai diffusa nel Milanese: «i pensieri della guerra esser più pressanti: sed belli graviores esse curas» (p. 1206).

Fortissima è infine, secondo il Manzoni, la responsabilità dei dotti nel non saper riconoscere, a parte alcune luminose eccezioni, la natura e la gravità del contagio. Certo anche il popolino credeva che la peste si diffondesse attraverso le pratiche magiche e gli untori, ma, precisa l'autore:

D'ugual valore, se non in tutto d'ugual natura, erano $i$ sogni [nostro il corsivo] de'dotti; come disastrosi del pari n'eran gli effetti (p. 1220).

Mi sembra evidente l'allusione al fatto che i dotti non seppero capire la natura del contagio e contribuirono anch'essi a diffondere la credenza negli untori, con i conseguenti processi e la condanna di innocenti, che solo la malafede interessata dei giudici poteva ritenere responsabili della diffusione della peste ${ }^{6}$. Persino l'amato e illuminato cardinale Federigo Borromeo credeva nelle unzioni. Cita, infatti, e traduce implacabile il Manzoni dal De Pestilentia del cardinale:

«Era opinion comune [...] che di questi unguenti se ne componesse in vari luoghi, e che molte fossero l'arti di metterlo in opera: delle quali alcune ci paion vere [nostro il corsivo], altre inventate» (p. 1222).

Non vi è dubbio che nel fosco panorama che abbiamo tracciato vi sia qualche eccezione, qualche caso, cioè, di dotto o potente che mette la propria cultura al servizio degli umili. Il Borromeo fonda la Biblioteca Ambrosiana (per quanto ovviamente inaccessibile agli analfabeti, la stragrande maggioranza della popolazione) e dispone, cosa eccezionale per l'epoca, che:

i libri fossero esposti alla vista del pubblico, dati a chiunque li chiedesse, e datogli anche da sedere, e carta, penne e calamaio, per prender gli appunti che gli potessero bisognare (p.1131).

Inoltre, almeno nella finzione narrativa, egli ha un abboccamento con Lucia, «miracolosamente» liberata dall'innominato, e con la madre Agnese, e si adopera per aiutare veramente la giovane. Forse ancora più chiaro l'esempio di padre Cristoforo, che mette

${ }^{6}$ È noto che la problematica del processo agli untori e delle sue implicazioni morali assunse sempre maggiore importanza nella considerazione del Manzoni, tanto da indurlo a pubblicare, in appendice alla Quarantana, il pamphlet intitolato alla Storia della colonna infame. 
la sua predicazione e la sua azione prima al servizio di Lucia perseguitata da don Rodrigo e poi degli appestati nel lazzeretto. Ma si tratta, appunto, di eccezioni, anche numericamente, nel complesso del romanzo, al quale è sottesa la stessa Weltanschauung giansenisticamente pessimistica che emerge dalle parole di Adelchi morente: «non resta / che far torto o patirlo» (Adelchi, atto V, vv. 351-52), qui solo attenuata dall'impegno personale di individui eccezionali come fra Cristoforo e il Borromeo.

Non a caso, l'ultimo esempio di latino (già lo si è visto, lingua per antonomasia della cultura) cui si accenna nella fabula del romanzo sono «certe paroline in latino» (p. 1271) con cui il matrimonio dei due umili protagonisti della storia viene suggellato dall' L'immancabilmente pavido don Abbondio, ma solo quando egli è assolutamente sicuro che don Rodrigo è passato a miglior vita, perché nel suo palazzotto è arrivato «l'erede per fidecommisso», il «signor marchese $* * *$ » (p. 1269), e dopo che egli ha esposto la sua teoria della peste come scopa, la quale

ha spazzato via certi soggetti, che, figliuoli miei, non ce ne liberavamo più: verdi, freschi, prosperosi: bisognava dire che chi era destinato a far loro l'esequie, era ancora in seminario, a fare i latinucci (p. 1270).

Due personaggi, don Abbondio e il marchese, l'ecclesiastico e il nobile, che, dopo la cerimonia nuziale, conversano con gli sposi e aiutano a servirli a tavola, ma poi si ritirano a pranzare da soli in un'altra stanza, poiché hanno tanta umiltà

quanta ne bisognava per mettersi al di sotto di quella buona gente, non per istar loro in pari (p. 1273).

Si osservi infine, a conclusione di questa analisi del rapporto fra gli umili e la cultura nei Promessi Sposi, che i personaggi storici del romanzo, la monaca di Monza, l'innominato, il cardinale (per non citare che i più celebri e più profondamente radicati nell'immaginario collettivo degli italiani) sono tutti di estrazione sociale elevata, mentre gli umili protagonisti il Manzoni se li è dovuti «inventare», anche se documentandosi sulle fonti storiche e secondo il principio della verosimiglianza teorizzato nella Lettre à $M$. Chauvet (p. 1671 ss.): ovviamente, perché Manzoni sapeva bene che la Storia la scrivono i vincitori, ed essa non si occupa degli umili e dei derelitti, ai quali si può semmai arrivare, pur partendo dalla verità della storia, solo attraverso la verità della poesia.

\section{I «VINTI» DI ACI TREZZA}

Nella prefazione (datata Milano, 19 gennaio 1881) a quello che avrebbe dovuto essere il primo romanzo del progettato e incompiuto ciclo dei Vinti, Giovanni Verga definisce I Malavoglia

lo studio sincero e spassionato del come probabilmente devono nascere e svilupparsi nelle più umili condizioni, le prime irrequietudini pel benessere ${ }^{7}$.

7 VERGA, Giovanni (1985): I Malavoglia, a c. di S. Guglielmino, Milano, Principato, p. 3. Da questa edizione sono tratte tutte le successive citazioni verghiane. 
Poco dopo, a ribadire la sua volontà di essere un narratore positivisticamente e scientificamente impersonale, egli aggiunge:

bisogna seguire scrupolosamente le norme di questa analisi (p.4),

concludendo poi:

Chi osserva questo spettacolo non ha il diritto di giudicarlo; è già molto se riesce a trarsi un istante fuori del campo della lotta per studiarla senza passione (pp. 5-6).

È noto come nelle sue opere veristiche il Verga persegua il canone dell'impersonalità attraverso l' «artificio della regressione ${ }^{8}$ : il narratore, cioè, adotta nei Malavoglia livelli e parametri di giudizio e di linguaggio «bassi», «regrediti» appunto rispetto a quelli che sarebbero propri di un intellettuale borghese qual è l'autore Verga, e che vogliono invece essere mimetici della mentalità e del parlato degli umili abitanti del paese catanese di Aci Trezza, dove è ambientata la vicenda narrata. Ciò non toglie che dal romanzo emerga chiaramente l'ideologia conservatrice e socialmente immobilistica del Verga, il quale non crede nel progresso storico e che, con i Malavoglia e la successiva novella Libertà, anticipa tutta una serie di romanzi storici che affrontano la cosiddetta questione meridionale e la delusione degli ideali risorgimentali dopo la proclamazione dell'unità d'Italia, il 17 marzo $1861^{9}$.

Proprio quest'ultimo tema si ricollega al rapporto tra gli umili e la cultura. Nei $M a$ lavoglia si possono individuare due «culture»: da un lato quella immobilistica ma al tempo stesso sanamente improntata all'etica del lavoro, della famiglia e dell'onestà, che padron 'Ntoni, analfabeta come tutti i Malavoglia e la stragrande maggioranza degli abitanti di Aci Trezza, esprime oralmente con i proverbi, «Perché il motto degli antichi mai mentì" (p. 8) ${ }^{10}$; dall'altro quella ufficiale e scritta, di cui sono depositari i notabili e gli «intellettuali» del paese, tutti personaggi per diversi aspetti negativi, da don Silvestro, corrotto e truffaldino segretario comunale, oltre che insegnante elementare, al vicario «reazionario» don Giammaria, perennemente in lite con lo speziale «rivoluzionario» don Franco, uno dei personaggi più ridicolizzati dal narratore ${ }^{11}$.

Ora, la vicenda dei Malavoglia comincia nel dicembre del 1863, all'indomani dell'unità nazionale italiana, quando 'Ntoni viene chiamato per la leva di mare ${ }^{12}$. Fin da sùbito, alla sincera disperazione, anche economica, del vecchio padron 'Ntoni( «Il re faceva così, che i ragazzi se li pigliava per la leva quando erano atti a buscarsi il pane», p. 14),

\footnotetext{
${ }^{8}$ Su cui diffusamente BALDI, Guido (1980): L'artificio dellla regressione. Tecnica narrativa e ideologia nel Verga verista, Napoli, Liguori.

${ }^{9} \mathrm{Si}$ veda a questo proposito NAVARRO SALAZAR, María Teresa (2000): «De la novela risorgimentale a la narrativa histórica italiana contemporánea», in Novela histórica europea, Madrid, UNED, pp. 66-70

${ }^{10} \mathrm{E}$ che, sia detto fra parentesi, non è del tutto sovrapponibile alla logica duramente economica e utilitaristica che regola i rapporti fra tutti gli abitanti del villaggio.

${ }^{11} \mathrm{Nel}$ crearlo, il Verga si ricordò di Homais, il farmacista «mangiapreti» di Madame Bovary, cui Gustave Flaubert contrappone l'ottuso e rigido Bournisien.

${ }^{12}$ La coscrizione obbligatoria è per gli umili pescatori di Aci Trezza il primo e tangibile segno negativo dell'Italia unita.
} 
si contrappone la reazione, per diversi aspetti interessata, dei tre personaggi sopra citati. Don Giammaria, nostalgico del regno delle Due Sicilie,

gli avea risposto che gli stava bene, e questo era il frutto di quella rivoluzione di satanasso che avevano fatto collo sciorinare il fazzoletto tricolore dal campanile (p. 9) ${ }^{13}$.

Don Franco, repubblicano e socialista,

gli giurava fregandosi le mani che se arrivavano a mettere assieme un po' di repubblica, tutti quelli della leva e delle tasse li avrebbero presi a calci nel sedere (ibidem).

Forse ancora peggiore la reazione del maneggione don Silvestro, il quale assicura che:

con un certo gruzzoletto fatto scivolare in tasca a tale e tal altra persona che sapeva lui [credo: anche lui stesso!], avrebbero saputo trovare a suo nipote un difetto da riformarlo (ibidem).

Ma il tentativo di corruzione si rivela inutile, poiché «il ragazzo era fatto con coscienza, come se ne fabbricano ancora ad Aci Trezza» (p. 9), con il risultato che «si presero "Ntoni senza dire "permettete"».

A mo’ di appendice a questo episodio iniziale del romanzo, si aggiunga che, partito militare,

dopo un po' di tempo 'Ntoni aveva pescato un camerata che sapeva di lettere, e si sfogava a lagnarsi della vitaccia di bordo, della disciplina, dei superiori (p. 13).

Per scrivere a casa, insomma, l'analfabeta 'Ntoni è costretto a ricorrere ad un intermediario che sappia leggere e scrivere. Allo stesso modo, il Renzo manzoniano, ormai in salvo nel Bergamasco sotto il falso ma significativo nome di Antonio Rivolta, per fare avere sue notizie a Lucia e Agnese, deve confidarsi con un

segretario, perché il poverino non sapeva scrivere, e neppur leggere, nel senso esteso della parola, e se, interrogato di ciò, come forse vi ricorderete, dal dottor Azzecca-garbugli, aveva risposto di sì, non fu un vanto, una sparata, come si dice; ma era la verità che lo stampato lo sapeva leggere, mettendoci il suo tempo: lo scritto è un altro par di maniche (p. 1173).

Dal canto suo, Agnese, per leggere la lettera di Renzo e rispondere,

trottò a Maggianico, se la fece leggere e spiegare da quell'Alessio suo cugino: concertò con lui una risposta, che questo mise in carta (ibidem).

Appena accennato dal Verga, e più ampiamente sviluppato con la consueta ironia dal Manzoni, la necessità di ricorrere a una persona «istruita» per scrivere lettere è evidentemente un tratto sociolinguistico che unisce, nello spazio e nel tempo, la Lombardia del Seicento alla Sicilia dell'Ottocento.

\footnotetext{
${ }^{13}$ Si osservi che con la stessa immagine inizia la novella Libertà.
} 
A proposito di legami fra i Malavoglia e i Promessi Sposi, a volte minimi, ma che denotano, come non potrebbe essere diversamente, una lettura attenta del capolavoro di Manzoni da parte del Verga, soffermiamoci un attimo sul dottor Scipioni, l'avvocato a cui, su consiglio di don Silvestro, i Malavoglia si rivolgono nel timore che, per il debito contratto con lo zio Crocifisso e che questi ha finto di vendere a Piedipapera, possa loro essere pignorata la casa del nespolo ${ }^{14}$. La figura del dottor Scipioni (il futuro Onorevole Scipioni del mai scritto quarto romanzo del ciclo dei Vinti?) è senza dubbio modellata su quella dell'Azzecca-garbugli manzoniano. Egli non è certo un imbroglione come il suo illustre predecessore, ma a lui sono indirizzati i Malavoglia «come i pulcini nella stoppa» (p. 88) ${ }^{15}$ da don Silvestro, cui, per ottenere consiglio sul da farsi, essi hanno fatto omaggio di «quelle due galline là» (p. 89) ${ }^{16}$. L'avvocato dice ai Malavoglia di non preoccuparsi per la casa, poiché su di essa grava l'ipoteca dotale di Maruzza, che non c'entra nulla con il debito contratto da padron 'Ntoni con lo zio Crocifisso. Sennonché, dietro subdolo consiglio di don Silvestro, Maruzza rinuncia all'ipoteca dotale sulla casa e alla fine i Malavoglia devono andarsene dalla casa del nespolo. Ora nemmeno l'abile avvocato Scipioni né può né vuole più fare nulla per padron 'Ntoni:

gli rideva sul naso, e gli diceva che «chi è minchione se ne sta a casa», che non doveva lasciarvi mettere la mano alla nuora, e poiché aveva fatto il pasticcio se lo mangiasse" (p. 148).

Il povero vecchio ritornerà dall'avvocato quando il nipote 'Ntoni verrà arrestato per avere ferito il brigadiere don Michele, che lo ha sorpreso in un' azione di contrabbando. Questa volta il giovane ma bravo avvocato, «quello delle chiacchiere» (p.265), che «si contentava di venticinque lire» (p. 89) per le sue consulenze ${ }^{17}$,

dopo che ebbe udito ogni cosa, e si fu raccapezzato [...] disse che era una bella causa, da buscarsi la galera, se non c'era lui, e si fregava le mani (p. 265).18

L'avvocato Scipioni accetterà di difendere 'Ntoni e, per fargliela «cavare con quattro o cinque anni di prigione» (p. 265), insisterà su una presunta questione d'onore. Accoltellando don Michele, 'Ntoni avrebbe voluto vendicare l'onore della sorella Lia:

don Michele se la intendeva con la Lia, la sorella di 'Ntoni Malavoglia, e ronzava là da quelle parti della strada del Nero tutte le sere per la ragazza. L'avevano visto anche quella notte della coltellata! (p. 270).

${ }^{14}$ Un albero di fico si trova, invece, nel cortiletto antistante la casa di Agnese e Lucia nei Promessi Sposi.

15 La stessa espressione ricorre nei Promessi Sposi in bocca ad Agnese (p. 969).

${ }^{16}$ La variante verghiana di «quei quattro capponi» che Renzo porta in omaggio all'Azzecca-garbugli, poiché, spiega Agnese, «non bisogna mai andar con le mani vuote da que' signori» (p. 969).

${ }_{17}$ Mutatis mutandis, è la stessa somma che Tonio deve a don Abbondio nei Promessi Sposi.

18 È la stessa sicumera con cui il manzoniano Azzecca-garbugli, anche lui in difficoltà a decifrare l'arruffata esposizione del suo incolto cliente, risponde a Renzo, da lui scambiato per un bravo che avrebbe minacciato un sacerdote perché non celebri un matrimonio: «Caso serio, figliuolo; caso contemplato. Avete fatto bene a venire da me», concludendo poco dopo: «Il caso è serio; ma voi non sapete quel che mi basti l'animo di fare, in un'occasione» (p. 971). 
Si tratta di una difesa giuridicamente valida, ma, dal punto di vista dell'etica dell'onore, propria dei Malavoglia, disastrosa: padron 'Ntoni ne avrà una crisi da cui non si riprenderà più, e la Lia per la vergogna «uscì nel cortile e poscia nella strada, e se ne andò davvero, e nessuno la vide più» (p. 271).

Se l'efficace professionalità dell'avvocato è indifferente ai valori dell'umile collettività di Aci Trezza, essa è almeno esente dalla malafede e dall'opportunismo dei tre «intellettuali» paesani citati all'inizio: don Silvestro, don Franco e don Giammaria.

Qui si accennerà appena al vicario Giammaria, ecclesiastico conservatore e contrario all'unità d'Italia, figura secondaria nello stesso romanzo. Nonostante le «parole latine» che «sputava in faccia» (p. 23) all'eterno antagonista don Franco, egli sembra mosso, se non ad agire, a parlare, più dall'astio verso il neonato Stato italiano o dalla stizza verso il laico don Franco e «i tempi e gli uomini nuovi» (p.187), o ancóra dal rancore personale per il denaro rubato prima a lui dalla sorella e poi a questa da don Silvestro, che dalla volontà di assistere almeno spiritualmente i suoi parrocchiani ${ }^{19}$. Una delle poche volte che lo si vede in azione è durante l'epidemia di colera del $1867^{20}$. Egli si reca a benedire la salma di Maruzza la Longa, ma:

rimase sulla soglia, quando spruzzò l'acqua santa coll'aspersorio, tenendo raccolta e sollevata la tonaca di san Francesco, - da vero frate egoista qual era! - predicava lo speziale (p. 202).

L'ottica malevola del commentatore non credo sminuisca la verità che don Giammaria è più attento a salvare la vita che ad adempiere ai doveri del suo ministero.

Più impietosamente e diffusamente tratteggiato nel corso di tutto il romanzo è il farmacista, don Franco. Il Verga non perde occasione per mettere in luce aspetti ridicoli o meschini del personaggio: dai particolari fisici (la pancia, lo «sballottarsi in cima alle gambette») alla costante paura della moglie, la Signora, alla presenza della quale egli non osa fiatare. Al suo estremismo verbale di divulgatore radical-socialista e di repubblicano anticlericale si contrappone, con chiari intenti di ridicolizzazione, la meschinità e la vigliaccheria dei comportamenti. A questo proposito, bastino due esempi. A p. 222 si dice che:

Lui non aveva difficoltà di starsene in sinedrio anche con quelli senza scarpe, purché non mettessero i piedi sui regoli delle scranne.

Poi, quando 'Ntoni manifesta il proposito di ribellarsi alle prepotenze del brigadiere don Michele,

Bravo! Esclamava lo speziale, così va fatto! Bisogna che il popolo mostri i denti. Ma lontano di qua, ché non voglio pasticci nella mia spezieria (p. 243) ${ }^{21}$.

19 È insomma la versione verghiana del don Abbondio di Manzoni.

${ }^{20}$ Del 1630 è invece l'altrettanto storica peste milanese dei Promessi Sposi.

21 Nei Promessi Sposi, secondo l'oste del paese di Renzo, i galantuomini sono «Quelli che [...] se hanno una coltellata da consegnare a uno, lo vanno ad aspettar fuori, e lontano dall'osteria, tanto che il povero oste non ne vada di mezzo» (p. 1006). 
La stoccata finale al suo personaggio il Verga la dà nel cap. XV: spaventato dall'essere stato in tribunale «in mezzo a tutti quegli sbirri» (p. 283) come testimone al processo contro 'Ntoni, il «rivoluzionario» diventa un agnellino:

Ora lo speziale non teneva più cattedra; e quando veniva don Silvestro, andava a pestare i suoi unguenti nel mortaio, per non compromettersi [...] E si sfogava soltanto con don Giammaria, e con don Ciccio il medico (p. 284).

Infine, si è già accennato ad alcune delle malefatte di don Silvestro, personaggio rappresentato senza alcuna connotazione comica o ironica. Segretario comunale, egli è l'effettivo detentore del potere, e il sindaco del paese, mastro Callà, detto significativamente «Giufà» (il tipo dello sciocco della tradizione popolare siciliana), non è altro che una marionetta nelle sue mani. Proprietario di vigne e delle «più belle chiuse di Trezza, - dove era venuto senza scarpe ai piedi - aggiungeva Piedipapera» (p. 23), egli «si beccava anche quel po' di stipendio di maestro elementare» (p. 30). Tutta la sua astuzia e tutta la sua cultura sono finalizzate al perseguimento di scopi personali non sempre del tutto limpidi. Ha un suo piano, sposare Barbara Zuppidda, e lo persegue con una sottile trama di alleanze e di mosse, non esitando a mettere i Malavoglia sulla via della rovina quando consiglia alla Longa di rinunciare all'ipoteca dotale. Non è certo un caso che, nel cap. VI, all'inizio di questa scena, si legga:

Don Silvestro, il quale sapeva di legge, stava passando il tempo costruendo una gabbia a trappola che voleva regalare ai bambini della Signora (pp. 91-2).

Il valore allusivo e simbolico di questa «gabbia a trappola» mi sembra evidente; e che alla fine Barbara Zuppidda si sposi invece con padron Fortunato Cipolla nulla toglie alla malignità delle trame del segretario comunale.

Nel cap. VII, quando scoppia la «rivoluzione delle mogli» in séguito alle notizie di nuove tasse ${ }^{22}$, don Silvestro, adeguando le sue mosse caso per caso, neutralizza i suoi oppositori: lo speziale ${ }^{23}$, la Zuppidda e la Betta, figlia di «Giufà». Espressione di una società in trasformazione, nella quale il potere deriva anche dagli affari, a volte loschi, dalle funzioni amministrative e dalla corruzione del sottogoverno ${ }^{24}$, don Silvestro è l'uomo nuovo», il self made man (si ricordi che è un «forestiero» arrivato ad Aci Trezza «senza scarpe ai piedi»), un eroe del proprio tempo, l'equivalente del don Calogero del Gattopardo di Tomasi di Lampedusa.

Attraverso la sua figura mi sembra che Verga realizzi la sua diagnosi più lucidamente negativa dei meccanismi dell'amministrazione pubblica, denunciando come il potere venga gestito dalla classe abbiente a proprio esclusivo vantaggio, e come si risolva in una pura finzione ogni principio di rappresentanza democratica ${ }^{25}$.

${ }^{22}$ Agli occhi dei popolani di Aci Trezza, il secondo aspetto negativo, dopo la coscrizione obbligatoria, dell'Italia unita.

${ }^{23}$ Di cui, ricordandosi di identica espressione di don Abbondio, egli dice che voleva «raddrizzare le gambe ai cani» (p. 23).

${ }^{24}$ Si è già visto, a proposito della visita di leva di 'Ntoni, che sapeva lui a chi fare «scivolare in tasca» un «certo gruzzoletto».

${ }^{25}$ Questa rappresentazione degli ingranaggi del potere, che si perpetua e cambia solo la facciata ma non la sostanza, è un tema che passerà da Verga al De Roberto dei Vicerè e al Tomasi del Gattopardo. 
Vorrei concludere sui Malavoglia con un significativo esempio dell'indifferenza degli apparati ufficiali del potere e della cultura con cui il potere si manifesta, verso la sorte degli umili, e, da un altro punto di vista, dell'estraneità delle classi subalterne al processo risorgimentale. Nel cap. IX, partito Luca per la leva di mare,

cominciò a correre la voce che nel mare verso Trieste ci era stato un combattimento tra i bastimenti nostri e quelli dei nemici, che nessuno sapeva nemmeno chi fossero, ed era morta molta gente (p. 144) ${ }^{26}$.

Siccome Luca non fa più avere notizie di sé, «il povero vecchio» padron 'Ntoni si decide ad andare a Catania con la nuora a chiedere informazioni del nipote:

Colà, dopo averlo rimandato per un pezzo da Erode a Pilato, si misero a sfogliare certi libracci e a cercare col dito sulla lista dei morti (p. 145).

A sottolineare la fredda indifferenza con cui viene comunicata la morte di Luca, il narratore ricorre all'ellissi e termina così l'episodio:

- Son più di quaranta giorni — conchiuse l'impiegato, chiudendo il registro. Fu a Lissa; che non lo sapevate ancora? (ibidem).

L'indifferenza dell'impiegato, per il quale sui «libracci» un nome vale l'altro, e il ritardo con il quale gli interessati apprendono la notizia della morte di un loro familiare, diventano emblema della lontananza e dell'estraneità dell'apparato statale rispetto all'esistenza degli umili.

\section{L'UTOPIA CONTADINA}

Vincitore del premio Supercampiello nel 1987, I fuochi del Basento di Raffaele Nigro si ricollega sia ai Promessi Sposi sia ai Malavoglia.

Al Manzoni, Nigro rinvia già nel risvolto di copertina, dove avverte che «I fatti narrati in questo libro sono (come sempre la Storia) un misto di cronaca e di immaginazione» ${ }^{27}$, affermazione che sembra ricalcata sulla definizione manzoniana del romanzo storico come «componimento misto di storia e d'invenzione». Poco dopo, egli cita le fonti storiche da cui ha tratto «notizie e suggestioni», aggiungendo che:

I registri dei rei di Stato degli Archivi di Potenza, di Matera e di Bari e della Regia Dogana di Foggia mi hanno suggerito una rivisitazione del concetto di non-storia formulato da Ernesto De Martino (ibidem).

${ }^{26}$ Il lettore sa che si tratta della battaglia di Lissa, combattuta il 20 luglio 1866 nel corso della terza guerra d'indipendenza.

27 NIGRO, Raffaele (2008): I fuochi del Basento, Milano, BUR, p. 24. Da qui anche tutte le citazioni successive. 
Insomma, Nigro denuncia fin da subito le sue fonti storiche, che invece il Manzoni, dopo avervi accennato nell'Introduzione, chiama a testimonianza in modo costante lungo tutto il corso del romanzo. Ma non è solo questo: citando il nome dell'etnologo napoletano Ernesto De Martino, profondo conoscitore del folklore lucano ed erede del metodo storico delle Annales francesi, il Nigro intende dare voce a quegli umili, a quei «vinti» di cui normalmente non si parla ${ }^{28}$. Emblematica in questo senso una frase di don Tommaso Bindi. A padre Raffaele Arcangelo, che, ricordandogli il suo precario stato di salute, cerca di distoglierlo dall'organizzare una nuova rivolta dei «cafoni senza terra», dopo la sconfitta dei moti del 1820-21, l'indomito Bindi risponde:

Nessuno ricorda mai gli sconfitti. Ma ogni tanto si affaccia un Giannone, un Cuoco e fa giustizia. Cent'anni dopo i fatti, magari”' (p. 281).

Lo si potrebbe definire il «sugo di tutta la storia», e certo denuncia la volontà dell'autore di riportare alla luce la microstoria dimenticata del popolo, così come Manzoni intende nei Promessi Sposi dare voce alla moltitudine di uomini che passa sulla sua terra senza lasciare traccia ${ }^{29}$. E come il Manzoni mescolava nelle sue pagine personaggi storici e personaggi d'invenzione, così anche il Nigro inserisce le vicende di cinque generazioni di omonimi contadini (senza terra!) nel piu vasto quadro storico del regno delle Due Sicilie, dall'esperienza della repubblica partenopea (1799) alla spedizione dei mille (1860): uno scenario in cui, accanto a Murat, al cardinal Ruffo e a Garibaldi, agisce, oltre ai Nigro, tutta una miriade di umili senza nome, unicamente riscattati dall'onomastica «barocca» e fiorita dell'autore ${ }^{30}$.

D'altra parte, si diceva che i Fuochi del Basento si ricollegano ai Malavoglia, nell'affrontare, sia pure in un'ottica novecentesca, la medesima questione risorgimentale e meridionale: sia il Nigro sia il Verga rappresentano il processo di unificazione nazionale dal punto di vista delle plebi meridionali, per le quali non molto cambia nel passaggio del potere dai Borboni ai Savoia. Ma senza «scadere», né l'uno né l'altro, da un punto di vista linguistico, nel dialetto, anche se in entrambe le opere forte è il peso del parlato regionale $^{31}$.

Non è qui possibile dar conto in modo dettagliato di tutta la saga della famiglia Nigro, ma si considereranno almeno le principali vicende collegate a tre personaggi particolarmente importanti per il rapporto degli umili con la cultura. A cominciare da Francesco Nigro, il «cantastorie» che proprio con la sua poesia ha conquistato il cuore della

${ }^{28}$ Lo ha ben visto NAVARRO SALAZAR, María Teresa (1996): «I fuochi del Basento o la metáfora del espacio como aproximación a la historia local», in La novela histórica a finales del siglo XX, José Romera et alii (Eds.), Madrid, Visor Libros, pp. 319-27.

${ }^{29}$ Secondo la celebre definizione del Discorso sopra alcuni punti della storia longobardica in Italia sopra citata.

${ }^{30}$ Resta da sottolineare (con NAVARRO SALZAR, 1996: 325-27) che la precisione topografica dello spazio geografico in cui si svolgono le vicende narrate è nel Nigro garanzia della veridicità delle stesse: nessuna falsificazione è possibile di fronte a chi conosce ogni angolo della «propria» terra.

31 A questo si aggiunge nel Nigro un tono epico e da cantastorie, che affonda le sue radici nell'arcaica cultura popolare lucana, a cui ha dato per la prima volta diritto di cittadinanza nella letteratura italiana il Cristo si è fermato a Eboli di Carlo Levi nel 1945. Per una rapida panoramica della letteratura lucana, si veda SPINELLI, Tito (1987): Letteratura delle regioni d'Italia. Storia e testi. Basilicata, Brescia, La Scuola. 
moglie Concetta Libera Palomba. Oltre alla poesia orale, egli ama anche la lettura, almeno da quando, nella masseria dei Galiani, dove lavorano i Nigro, trova il quinterno di un libro. Da allora desidera imparare a leggere libri e, sulla tomba del figlio Pietropaolo, scommette che, se nasceranno ginestre sulla sua sepoltura, potrà sperare di comprendere prima i segni tracciati sui libri e poi imparare a tracciarne anch'egli di simili (p. 31). Padre di numerosi figli, l'ultimo dei quali è Raffaele Arcangelo (v. infra), quando la situazione storica vede i francesi alle porte del regno di Napoli, Francesco Nigro si dà alla macchia ed entra nella banda del Cidognese, che lo mette súbito alla prova del fuoco e del sangue. Di essa, la storia ufficiale sa che «avvenne sotto Canosa, sulla via Appia antica, nei pressi di alcune fabbriche distrutte dai frequenti terremoti» (p. 54). Partendo da questa scarna informazione degli archivi dei potenti, il Nigro «manzonianamente» ricostruisce i sentimenti e le azioni di Francesco: soprattutto la notizia che, mentre gli altri briganti si gettano sull'oro e sulle donne, egli chiede per sé, come parte del bottino che gli tocca, libri altrimenti destinati a diventare cenere, «una scelta da uomo e non da contadino» (p. 57). Ben presto, poi, Francesco prende le parti dei braccianti in lotta contro i soldati regolari che li stanno facendo a pezzi e incontra don Tommaso Bindi, che gli parla delle teorie di Rousseau e della rivoluzione, da cui nasceranno una nuova classe dirigente, "pane per tutti e amnistia ai reati" (p. 65). Così, da brigante Francesco Nigro diventa generale e non combatte più per arricchirsi, ma lotta per l'emancipazione dei contadini: «uccide per migliorare il mondo, per il bene della povera gente e Dio giustifica» (p. 106); e nella grotta del suo quartier generale raccoglie una biblioteca con cui sogna di riempire la sua futura casa.

Gli eventi però incalzano e la reazione sanfedista e borbonica insanguina il regno, contapponendo gli uni agli altri non solo gli uomini, ma anche diverse concezioni di Dio: il Dio del cardinal Ruffo e del re versus il Dio dei giovani intellettuali giacobini e dei contadini, il Dio delle gerarchie versus il Dio dell'uguaglianza. Tale contrapposizione è ben resa nelle pp. 130-32 del romanzo, alla vigilia della battaglia di Picerno, dove Francesco incontrerà la morte. Prima della battaglia avviene l'incontro-scontro fra i comandanti dei due opposti schieramenti, Francesco Nigro e Gerardo Curcio ${ }^{32}$. Nella mente di Curcio vi è la convinzione che «Le dita della mano non sono uguali, e l'ordine delle leggi è simile all'ordine della natura» $(\text { p. 132 })^{33}$. Viceversa, il Dio di Francesco Nigro è lo stesso di Tommaso Bindi, «è il diritto che vuole gli uomini tutti uguali [...] è la ragione [...] è l'energia di quelli che vogliono imparare a leggere, a scrivere, a capire» (p. 128), perché un altro Francesco non sia più costretto a ricorrere ad un interprete per poter leggere la lettera di un altro Tommaso, come invece succede qui (vedi p.126) ${ }^{34}$.

Siamo così arrivati al secondo personaggio che ci interessa, don Tommaso Bindi, depositario di una cultura che lotta per il riscatto degli umili e l'uguaglianza tra gli uomini. È lui a donare a Francesco Arcangelo il terreno su cui costruire:

32 «Un contadino [...] che nega le sue origini per gli interessi dei baroni che lo hanno tenuto schiavo», secondo le sprezzanti parole di Tommaso Bindi (p. 127).

${ }^{33} \mathrm{Si}$ osservi che, seppure in altro contesto, anche padron 'Ntoni pensa che «Gli uomini son fatti come le dita della mano: il dito grosso deve far da dito grosso, e il dito piccolo deve far da dito piccolo» (I Malavoglia, pp. 7-8).

${ }^{34}$ Un tema, questo dell'incapacità di leggere e scrivere lettere, che, come si ricorderà, abbiamo già incontrato sia nei Promessi Sposi sia nei Malavoglia. 
un ospedale, un ospizio per poveri, malati, vagabondi, trovatelli. Un regno per i disgraziati, o se vi piace, una repubblica del Preziosissimo Sangue, a difesa del corpo e dello spirito (p. 288).

Inoltre si premura di mettere nero su bianco:

Tanto io non faccio secondo consuetudine ecclesiale e more articuli mortis, ma per difesa dei bisognosi e degli infermi (ibidem) ${ }^{35}$.

L'illuminista Tommaso Bindi non smette mai di sognare, pur riconoscendo il carattere utopico del suo progetto, una repubblica di contadini ${ }^{36}$, e crede che fondamentale per la realizzazione di questo ideale sia la cultura. Non a caso «Il salotto verde di casa Bindi era dominato dai libri, come d'altro canto lo erano i saloni i corridoi lo studio" (p. 267); e il padrone di casa, dopo essersi allontanato dal Circolo dei Galantuomini, «ciucci che giocano a scacchi», «scriveva e leggeva» (p. 250). Certo, nonostante la presenza in essa anche del Metastasio ${ }^{37}$, la biblioteca di un uomo così, che «al tempo della grande diatriba tra gli antichi e i moderni si era schierato per D'Alembert, Voltaire, Diderot, Louis Blanc e Rousseau» e che «Dei napoletani ammirava Vico e cominciava a nutrire rispetto per Galiani e i suoi trattati economici» (p. 250), noi ce la immaginiamo ben diversa da quella piena di vacue auctoritates del don Ferrante di manzoniana memoria. Non credo infine che sia del tutto secondaria la precisazione che all'economia egli si avvicina quasi per caso, sfogliando i registri contabili della casa, meticolosamente tenuti dalla moglie, donna Annalisa Manna ${ }^{38}$.

Tommaso Bindi sogna un governo di intellettuali e contadini («Scrivani e contadini», p. 256), e io credo che la sua amicizia con Francesco Nigro simboleggi quella che NAVARRO SALAZAR(1996: 324) definisce «La unión entre intelectuales y campesinos enfrentados a la nobleza de terratenientes». Solo su queste basi si intende il valore non solo poetico ma anche politicamente emblematico dell'incontro post mortem fra i due, al cancello del cimitero, alla presenza di «una diecina di uomini, tutti vestiti di bianco, pagliette in testa e scalzi», i quali, come Francesco Nigro spiega a Tommaso Bindi, «Vengono a rendervi omaggio per il grande amore che gli avete dedicato» (p. 294).

Se Francesco Nigro, nonostante il suo amore per i libri, rimane analfabeta, l'ultimo dei suoi figli, Raffaele Arcangelo, è invece destinato ad altre esperienze di vita. Carmelitano scalzo, il giovane si forma una cultura sulle vite dei santi, ma al tempo stesso, a contatto con i poveri, accumula «una lunga esperienza della morte per fame, per malattia e vecchiaia» (p. 220). Ricevute le stimmate, dopo le prime informazioni sul padre che gli dà la madre, Raffaele Arcangelo si reca per saperne di più da Tommaso Bindi, il quale significativamente gli dice di Francesco Nigro:

35 Finalmente un latino che non è latinorum ma competenza giuridica illuministicamente piegata a favore dei poveri.

36 «Ci vuole tempo e pazienza, re Nasone era amante dei nobili di sangue, re Gioacchino ama i galantuomini e i massari. Chissà se verrà mai un governo disposto a elevare i servi» (p. 213).

37 Che gli serve per chiudere un'arringa con «una cosa comica» (p. 252).

${ }^{38}$ Il fatto è che le donne hanno in questo romanzo un ruolo nient'affatto secundario, se anche solo si pensa che, in un contesto molto più povero, anche Concetta Libera Palomba, vera e propria mater familias, gestisce la casa in assenza del marito Francesco. 
Non ci sono parole per ricordarlo [...] Ma questo può dirti tutto: non sapeva leggere, eppure aveva accumulato nelle grotte di Monticchio più libri di questa biblioteca; uno così, non è un uomo che fa meraviglia?" (p. 253).

È così che Raffaele Arcangelo, come il padre, si incontra con le esperienze e le letture di don Tommaso Bindi, e ciò lo spinge a ripensare con occhi diversi alla conoscenza, alla scienza e alla missione di un credente nel mondo dei vivi. Quindi, con l'aiuto del testamento di don Tommaso Bindi, innalza la Casa del Preziosissimo Sangue, dove, richiamandosi alla regola di povertà di san Francesco d'Assisi, egli, insieme ai suoi «confratelli», ${ }^{39}$ si prefigge di "sfamare gli affamati, radunare gli orfani abbandonati sulle strade, combattere l'ignoranza e la superstizione" (p.306). Ma non tutti nella Chiesa sono d'accordo con il suo proselitismo, e, quando Raffaele Arcangelo viene accusato di superbia e di sollecitare il fanatismo degli ingenui, solo l'intelligenza di monsignor Giovanni Bovio ${ }^{40}$, non a caso anche «uomo di studi profondi e di profonde letture» (p. 315) lo salva e gli consente di rimanere nella Casa.

Di nuovo, però, la storia travolge tutto: dopo l'unificazione, il brigante Pietro Senzanaso e la sua banda vengono sorpresi da uno squadrone di cavalleggeri piemontesi nei pressi della Casa di Raffaele Arcangelo e vi si rifugiano per meglio difendersi. Ne nasce una breve ma cruenta battaglia, che coinvolge anche gli abitanti della Casa e lo stesso padre Raffaele, che viene falciato alla prima scarica di fucileria.

Così termina la vita terrena di questo alter Christus; ma io vorrei continuare con due osservazioni. La prima riguarda il brigante Senzanaso, originariamente bracciante nelle terre del principe Torella, il quale, in una pagina di amara ironia (356-7), spiega quale bel passo in avanti sia stato, per lui ed i suoi compagni di fatica, passare da un padrone lontano ai soprastanti, che gli stanno sul collo e vivono, allo stesso modo dei padroni di prima, sfruttando il suo sudore: questo è, per lui e per molti altri diseredati del Sud, l'unico risultato della spedizione di Garibaldi e dell'unità d'Italia ${ }^{41}$.

Merita infine di essere ricordata l'osservazione che fa il caporale Botta, piemontese, prima di andarsene dopo la sepoltura di padre Raffaele Arcangelo:

Ho conosciuto a Torino un uomo come questo. Si chiama Giovanni Bosco. Fonda ospizi e orfanatrofi (p. 359).

Insomma, Nord e Sud uniti non solo nell'analfabetismo di chi, a diverse latitudini e in diverse epoche, ha bisogno di interpreti per leggere e scrivere lettere, ma anche nell'impegno di uomini di cultura e di fede che lottano perché le cose cambino, poiché, secondo le parole dello stesso Raffaele Arcangelo, «Dobbiamo riconquistare il miele della conoscenza e della scienza» (p. 265).

Vorrei aggiungere un'ultima osservazione riguardante il finale dei tre romanzi. Essi si concludono tutti con un «esilio»: nei Promessi Sposi, Renzo e Lucia se ne vanno dal

${ }^{39}$ Che continuano però a portare gli abiti di sempre, a significare che sono «uomini in mezzo agli uomini» (p. 306).

${ }^{40}$ Lo definirei un nuovo cardinal Borromeo.

${ }^{41} C f r$. su questo tema quanto si è detto prima a proposito dei Malavoglia. 
paese nativo e dai loro «monti» ed emigrano nel Bergamasco; nei Malavoglia, 'Ntoni, che ha rifiutato «l'ideale dell'ostrica», sente di essere ormai estraneo alla vita di Aci Trezza e se ne allontana sconsolato; infine, la nota conclusiva dei Fuochi del Basento non solo informa della partenza di Bartolomeo Nigro per l'America, ma, collegando il tempo del racconto al presente della cronaca, aggiunge:

Nel nostro secolo, all'inizio degli Anni Cinquanta, la Riforma fondiaria esaudì in parte le antiche richieste dei braccianti e divise in quote il patrimonio demaniale e latifondiero. Gli assegnatari vendettero le quote e fuggirono verso le città del Nord" (p. 362).

Ora, a me sembra che questi tre finali abbiano sì qualcosa in comune, ma al tempo stesso presentino ciascuno una propria specificità. Infatti, è senz'altro vero in generale quello che, nei Promessi Sposi, il Manzoni afferma per i suoi umili protagonisti, cioè che «le memorie triste, alla lunga guastan sempre nella mente i luoghi che le richiamano" (p. 1274), ma credo anche che vi sia un graduale venir meno di ogni speranza a mano a mano che ci si avvicina cronologicamente al presente di oggi. Così, secondo il cattolico Manzoni, da un lato «del dolore, ce n'è, sto per dire, un po' per tutto» (ibidem), dall'altro però non viene meno la speranza nell'aldilà, secondo le celeberrime parole conclusive dell'Addio, monti:

Chi dava a voi tanta giocondità è per tutto, e non turba mai la gioia de' suoi figli, se non per prepararne loro una più certa e più grande (p. 1018).

In Verga, se 'Ntoni si autoesclude da un mondo i cui valori egli ha in precedenza rinnegato, è però vero che la vita ad Aci Trezza continua, anche dal punto di vista del bighellone Rocco Spatu, che, pur nel suo ruolo di personaggio negativo, dal villaggio non si è mai allontanato né mai si allontanerà; anzi, come recita il finale, «il primo di tutti a cominciar la sua giornata è stato Rocco Spatu» (p. 297).

Viceversa, il Nigro, dimenticata almeno nel finale ogni prospettiva ultraterrena, conclude che, dopo la morte del fondatore, la Casa del Preziosissimo Sangue «Diventò terreno incolto tra terreni incolti» (p. 359) e registra l'esodo dei contadini dalle terre loro finalmente assegnate verso il miraggio dell' America o delle città del Nord. Le traversie dei nuovi immigranti che vengono ora da noi a cercare, per dirla con le parole del regista Gianni Amelio, Lamerica, gettano pesanti dubbi sul fatto che nelle nuove realtà essi abbiano davvero incontrato quella felicità, quella giustizia, quella cultura che cercavano lasciando il loro paese.

\section{REFERENCIAS BIBLIOGRÁFICAS}

\section{BIBLIOGRAFIA PRIMARIA}

Manzoni, Alessandro (1973): Tutte le opere, a c. di Mario Martelli, Firenze, Sansoni, 1973, 2 voll. Nigro, Raffaele ([1987] 2008): I fuochi del Basento, pref. di Ettore Catalano, Milano, BUR. VERGA, Giovanni ([1891] 1985): I Malavoglia, a c. di Salvatore Guglielmino, Milano, Principato. 


\section{BIBLIOGRAFIA SECUNDARIA}

BALDI, Guido (1980): L'artificio della regressione. Tecnica narrativa e ideologia nel Verga verista, Napoli, Liguori.

FORTI, Fiorenzo (1981): «Manzoni e il rifiuto dell'idillio», in Lo stile della meditazione. Dante Muratori Manzoni, Bologna, Zanichelli, pp. 160-88.

NAvarro Salazar, María Teresa (1996): «I fuochi del Basento o la metáfora del espacio como aproximación a la historia local», en La novela histórica a finales del siglo XX, José Romera Castillo et alii (Eds.), Madrid, Visor Libros, pp. 319-27.

- (2000): «De la novela risorgimentale a la narrativa histórica italiana contemporánea», in AA.VV., Novela histórica europea, ed. María Teresa Navarro Salazar, Madrid, UNED, pp. 6378.

RaImond, Ezio (2000): Il romanzo senza idillio. Saggio sui Promessi Sposi, Torino, Einaudi.

Spinelli, Tito (1987): Letteratura delle regioni d'Italia. Storia e testi. Basilicata, Brescia, La Scuola. 